\title{
Retrospective non-inferiority study of preoperative bilirubin level on surgical outcomes in hepatectomy with hilar resection
}

\author{
Sunhawit JUNRUNGSEE*, Rabin KAMMANEE, Worakitti LAPISATEPUN, Anon CHOTIROSNIRAMIT, Wasana KO-IAM \\ Department of Hepatobiliary and Pancreatic Surgey, Faculty of Medicine, Chiang Mai University, Chiang Mai, Thailand
}

Introduction: Elevated preoperative bilirubin affects the morbidity and mortality significantly in biliary tract surgery. Routine preoperative biliary drainage has been suggested and bilirubin level has been kept lower than $3 \mathrm{mg} / \mathrm{dL}$ before a major hepatectomy with hilar resection. However, the serum bilirubin may affected with the undrained liver lobe. The target bilirubin level less than $3 \mathrm{mg} / \mathrm{dL}$ probably unnecessary in all patients.

Methods: To study the association of preoperative bilirubin level with post-operative complications and mortality.

Results: There were 51 and 30 patients in group A and B. The operative blood loss was higher in group B but there was no significant difference. The incidence of post hepatectomy liver failure was comparable in group A and B [3.92\% vs. 3.33\% $(p=0.898)$ ]. There was no significant different of 30-day mortality and 1-year survival between group A and B [3.92\% vs. 6.67\% $(p=0.624)$ and 76.47 vs. 73.33 $(p=0.536)]$. Multivariate analysis revealed significant factors that associated with mortality were post hepatectomy liver failure adjusted hazard ratio $=93.70(p<0.001)$.

Conclusions: The surgical outcome in patients who have preoperative total bilirubin level between 3 and $10 \mathrm{mg} / \mathrm{dL}$ are non-inferior to bilirubin less than $3 \mathrm{mg} / \mathrm{dL}$. Post hepatectomy liver failure is the independent factor that associated with postoperative mortality which relate to other factors such as future remnant liver volume or major complication. 